\title{
A Linear Temporal Increase in Thrombin Activity and Loss of Its Receptor in Mouse Brain following Ischemic Stroke
}

\section{Doron Bushi 1,2, Efrat Shavit Stein 1, Valery Golderman 1,2, Ekaterina Feingold 1,2, Orna Gera ${ }^{1,2,3}$, Joab Chapman ${ }^{1,2,4,5+}$ and David Tanne ${ }^{1,4 * t}$}

${ }^{1}$ Comprehensive Stroke Center, Department of Neurology, The J. Sagol Neuroscience Center, Chaim Sheba Medical Center, Tel HaShomer, Israel, ${ }^{2}$ Department of Physiology and Pharmacology, Sackler Faculty of Medicine, Tel Aviv University, Tel Aviv, Israel, ${ }^{3}$ Department of Physical Therapy, Sackler Faculty of Medicine, Tel Aviv University, Tel Aviv, Israel, ${ }^{4}$ Department of Neurology, Sackler Faculty of Medicine, Tel Aviv University, Tel Aviv, Israel, ${ }^{5}$ Robert and Martha Harden Chair in Mental and Neurological Diseases, Sackler Faculty of Medicine, Tel Aviv University, Tel Aviv, Israel

Background: Brain thrombin activity is increased following acute ischemic stroke and may play a pathogenic role through the protease-activated receptor 1 (PAR1). In order to better assess these factors, we obtained a novel detailed temporal and spatial profile of thrombin activity in a mouse model of permanent middle cerebral artery occlusion (pMCAo).

OPEN ACCESS

Edited by:

Ashfaq Shuaib,

University of Alberta, Canada

Reviewed by:

Henry Ma,

Monash University, Australia

Terence J. Quinn,

University of Glasgow, UK

*Correspondence:

David Tanne

david.tanne@sheba.health.gov.il

tThese authors have contributed equally to this work as last authors.

Specialty section:

This article was submitted to Stroke, a section of the journal

Frontiers in Neurology

Received: 10 January 2017

Accepted: 24 March 2017

Published: 10 April 2017

Citation:

Bushi D, Stein ES, Golderman V, Feingold E, Gera O, Chapman J and

Tanne D (2017) A Linear Temporal Increase in Thrombin Activity and Loss of

Its Receptor in Mouse Brain following Ischemic Stroke. Front. Neurol. 8:138.

doi: 10.3389/fneur.2017.00138
Methods: Thrombin activity was measured by fluorescence spectroscopy on coronal slices taken from the ipsilateral and contralateral hemispheres 2, 5, and $24 \mathrm{~h}$ following pMCAo ( $n=5,6,5$ mice, respectively). Its spatial distribution was determined by punch samples taken from the ischemic core and penumbra and further confirmed using an enzyme histochemistry technique $(n=4)$. Levels of PAR1 were determined using western blot.

Results: Two hours following pMCAo, thrombin activity in the stroke core was already significantly higher than the contralateral area (11 \pm 5 vs. $2 \pm 1 \mathrm{mU} / \mathrm{ml})$. At 5 and $24 \mathrm{~h}$, thrombin activity continued to rise linearly $(r=0.998, p=0.001)$ and to expand in the ischemic hemisphere beyond the ischemic core reaching deleterious levels of $271 \pm 117$ and $123 \pm 14 \mathrm{mU} / \mathrm{ml}$ (mean $\pm \mathrm{SEM}$ ) in the basal ganglia and ischemic cortex, respectively. The peak elevation of thrombin activity in the ischemic core that was confirmed by fluorescence histochemistry was in good correlation with the infarcts areas. PAR1 levels in the ischemic core decreased as stroke progressed and thrombin activity increased.

Conclusion: In conclusion, there is a time- and space-related increase in brain thrombin activity in acute ischemic stroke that is closely related to the progression of brain damage. These results may be useful in the development of therapeutic strategies for ischemic stroke that involve the thrombin-PAR1 pathway in order to prevent secondary thrombin related brain damage.

Keywords: ischemic stroke, thrombin, permanent middle cerebral artery occlusion, protease-activated receptor, endothelial protein $\mathrm{C}$ receptor

Abbreviations: $\mathrm{BBB}$, blood-brain barrier; EPCR, endothelial cell protein C receptor; NSA, 5-nitrosalicylaldehyde; OGD, oxygen-glucose deprivation; PAR1, protease-activated receptor 1; pMCAo, permanent middle cerebral artery occlusion; PR3, proteinase 3; TTC, triphenyl tetrazolium chloride. 


\section{INTRODUCTION}

The "time is brain" paradigm is now well established in acute stroke care and emphasizes that neuronal tissue is rapidly lost as stroke progresses (1). Similarly, in permanent middle cerebral artery occlusion (pMCAo) performed in rats, infarction in the ischemic core progresses rapidly and the ischemic damage expands to the peripheral region with time (2). Understanding the mechanisms underlying the propagation of damage during stroke progression is critical for targeting effective treatments that can minimize irreversible neuronal damage.

Thrombin is a coagulation factor and a target for prevention of cardioembolic stroke $(3,4)$. In addition to its role in thrombogenesis, thrombin has important pleiotropic effects through the activation of its major receptor, protease-activated receptor 1 (PAR1) $(5,6)$. Activation of this receptor by low concentrations of thrombin may have neuroprotective effects, while at higher concentrations thrombin has deleterious effects (7-11). In the setting of an acute ischemic stroke, high thrombin levels have been detected in the infarct area, possibly as a consequence of blood-brain barrier opening $(12,13)$ or as a result of its local synthesis in the brain $(14,15)$ or both. Functionally, thrombin has been shown to cause synaptic dysfunction (15-19) and later, on vascular disruption, inflammatory response and neuronal damage (20-22), through the activation of PAR1 $(13,20)$. Indeed, the toxic effects of thrombin in the central nervous system have been shown in various ischemia models where neuroprotection could be achieved by PAR1 deletion (22). Recently, it was reported in pre-clinical studies that the direct thrombin inhibitor, argatroban, is protective when it is given immediately or after 1,2,3, but not $4 \mathrm{~h}$ delay from the ischemic event $(23,24)$. Moreover, there are clinical data that suggest that thrombin inhibition may be effective not only in prevention of cardioembolic stroke but also in the context of acute stroke (25-27). In addition, Chen et al. showed that continuous administration of PAR1 antagonist immediately after MCA occlusion decreased the level of neurovascular injury (13). However, in studies of the PAR1 antagonist, Vorapaxar (Merck, USA), in patients with prior ischemic stroke, there was an increased risk of intracranial hemorrhage without an improvement in major vascular events (28). Thus, better understanding the role of thrombin and PAR1 progression following ischemic event may have significant inputs for the development of therapeutic strategies based on thrombinPAR1 pathway.

Thrombin also cleaves the zymogen protein $\mathrm{C}(\mathrm{PC})$ to its activated form (aPC). Effective activation of PC by thrombin requires the transmembrane glycoprotein, thrombomodulin (TM), as a cofactor. PC activation by the thrombin-TM complex is enhanced $\sim 20$-fold in vivo when $\mathrm{PC}$ is bound to the endothelial cell protein C receptor (EPCR) (29). Retention of aPC bound to EPCR allows aPC to express multiple cytoprotective activities that involve PAR1. These cytoprotective activities include aPC-mediated anti-inflammatory and anti-apoptotic activities, alterations of gene expression profiles, and protection of endothelial barrier functions (30-33). Recently, we reported that different concentrations of thrombin affect long-term potentiation (LTP) through different molecular routes converging on PAR1. High thrombin concentrations induce a slow onset LTP, whereas low concentrations of thrombin promote LTP through aPC-EPCR-mediated mechanism (18).

In order to examine the role of thrombin-PAR1-EPCR during stroke progression, we sought to determine the profiles in time and space of thrombin activity as well as the relevant PAR1 and EPCR levels in mice brains during pMCAo. Mapping thrombin activity throughout the brain provided the opportunity to assess both the ischemic core and its surrounding penumbral areas. This is of particular importance since the thrombin-PAR-1 pathway is an attractive target for the development of novel therapeutics for ischemic stroke $(13,19,22,23,34-36)$ and may potentially prevent secondary thrombin-related brain damage. Clarifying the temporal and spatial profiles of thrombin and PAR1 following an ischemic event is fundamental for determining therapeutic strategies based on this pathway.

\section{MATERIALS AND METHODS}

\section{Animals}

Studies were carried out on 8-week-old male C57BL6 mice (23-30 gram, Harlan Laboratories Inc., Israel). Mice were kept at the animal house in mouse cages (six/cage) with free access to food and water with a 12/12-h light-dark circle. Anesthesia was performed with $2.5 \%$ isoflurane mixed in oxygen and delivered through a facemask. This type of anesthesia is common for this stroke model. PMCAo was performed using the common filament model based on our previously reported technique (12), using silicone-coated filament (Doccol Corp, Redlands, CA, USA). Mice were sacrificed using $100 \mu$ pental by i.p. administration. Immediately prior to sacrifice, motor deficits were measured for each animal and scored using a 5-point Neurologic Severity Scores described as follows (37): 0 , no neurologic deficit; 1 , failure to extend left forepaw fully; 2 , circling to the left; 3 , falling to the left; 4 , depressed level of consciousness and failure to walk spontaneously. Mice were divided into three groups according to the sacrifice time: $2(n=5), 5(n=6)$, and $24(n=5) \mathrm{h}$ following pMCAo. Another group of healthy mice served as control $(n=5)$. Sample sizes were chosen based on the magnitude of thrombin changes in our previous experiences $(12,38)$. Exclusion criteria include massive bleeding during surgery and/or intracerebral hemorrhage.

\section{Thrombin Activity Assay}

Thrombin activity was measured using a fluorometric assay as described previously $(12,39)$. Briefly, thrombin activity was measured on fresh coronal slices taken from the ischemic and contralateral hemispheres by a fluorometric assay, quantifying the cleavage of the synthetic peptide substrate Boc-Asp(OBzl)Pro-Arg-AMC (I-1560 Bachem, Switzerland). Following sacrifice, the brain of each animal was immediately removed and placed in a steel brain matrix ( $1 \mathrm{~mm}$, Coronal; Stoelting; IL, USA). First, the brain was cut sagittally in its midline so the left contralateral and the right ischemic hemispheres were separated. Then, the brain was cut starting at its anterior side (starting at slice \# 3,2 mm anterior to the bregma), into coronal 1-mm thick slices. The slices were placed into black microplate (Nunc; Denmark) containing the substrate buffer. Measurements were performed microplate 
reader (Tecan; infinite 200; Switzerland) with excitation and emission filters of $360 \pm 35$ and $460 \pm 35 \mathrm{~nm}$, respectively. For calibration, known concentrations of bovine thrombin (SigmaAldrich, Israel) were used in the same assay.

\section{Distribution of Thrombin Activity Levels in Mouse Brain following pMCAo}

The spatial distribution of thrombin activity in the ischemic core (coronal slice \#6 as described in the previous section) was determined $24 \mathrm{~h}$ following pMCAo, using the thrombin activity assay and $1.5-\mathrm{mm}$ diameter tissue punches that were taken from the cortical and basal ganglia areas in the ischemic and contralateral hemisphere. Each tissue sample was placed in a separate well in the microplate, and thrombin activity was measured using the thrombin activity assay. Following removal of the punch tissue samples, the punctured slices were stained using triphenyltetrazolium chloride (TTC) for infarct assessment.

\section{Histochemical Visualization of Thrombin Activity in Mouse Brain following pMCAo}

In addition to the tissue punches technique, the spatial distribution of thrombin activity in mice brain following pMCAo was determined using a novel enzyme histochemical method for visualization of the thrombin activity location in brain slice that we have developed (38). Briefly, the method is based on the cleavage of the substrate, Boc-Asp(OBzl)-Pro-Arg-4MßNA by thrombin to liberate free $4 \mathrm{M} \beta \mathrm{NA}$ which, in the presence of 5-nitrosalicylaldehyde (NSA), is captured, yielding an insoluble yellow fluorescent product that marks the site of thrombin activity.

Twenty-four hours following pMCAo, mice were sacrificed and their brains were immediately removed and inserted into $30 \%$ sucrose solution for $24 \mathrm{~h}$ at $4^{\circ} \mathrm{C}$. Thereafter, the brains were cut into $20 \mu \mathrm{m}$ coronal slices using a cryostat (Leica CM1850, Leica, Germany), and the cut sections were picked up on microscopic glass slides (Superfrost Plus, Thermo Scientific, USA). For thrombin activity staining, the slices were incubated in a solution containing $93 \mu \mathrm{l}$ of thrombin buffer (50 mM TRIS/HCl, pH 7.0, $0.15 \mathrm{M}$ $\mathrm{NaCl}, 1 \mathrm{mM} \mathrm{CaCl} 2), 1 \mu \mathrm{l}(0.6 \mathrm{mM}) \mathrm{NSA}, 1 \mu \mathrm{l}$ of an aminopeptidase inhibitor bestatin $(0.1 \mathrm{mg} / \mathrm{ml}$, Cayman Chemical Company, USA) and $5 \mu \mathrm{l}(0.1 \mathrm{mM})$ of the thrombin substrate Boc-Asp(OBzl)-ProArg-4M $\beta$ NA (GL Biochem, Shanghai, China). The stained slices were placed at room temperature for $24 \mathrm{~h}$. The specificity of the method for thrombin activity was verified using sections that were incubated in the histochemical solution containing $60 \mu \mathrm{M}$ of the highly specific thrombin inhibitor, $\mathrm{N} \alpha$-(2-naphthylsulfonylglycyl)4-amidino-(D,L)-phenylalanine piperidide acetate (NAPAP, Pefabloc TH, Sigma-Aldrich, Israel). The reaction was terminated by rinsing the sections in cold $50 \mathrm{mM}$ TRIS/HCL, pH 7.0. Next, the sections were fixed using $4 \%$ paraformaldehyde for $20 \mathrm{~min}$, washed with PBS and $0.1 \%$ Triton X-100, and incubated for $10 \mathrm{~min}$ with Hoechst (1:1,000, hoe-33342, Sigma-Aldrich, Israel) for nuclear staining. Finally, the sections were air dried and closed with mounting media and cover glass. Thrombin activity localization and hoechst staining were visualized using inverted fluorescence microscope $(\mathrm{I} \times 81$, Olympus, Japan) with a filter cube U-MWU2 (BP 300-385, BA420, DM400, Olympus, Japan).

\section{Western Blot}

Following the thrombin activity assay, coronal slices numbers 5-7 from each hemisphere were pooled and homogenized using a pestle motor mixer (Argos Technologies, USA). Proteins from the brain homogenates were separated by polyacrylamide gel electrophoresis and transferred onto nitrocellulose membranes for western blot analysis. Membranes were incubated with primary rabbit anti PAR1 antibody (1:500, ABCAM-ab32611, Abcam, USA) and primary rabbit anti EPCR antibody (1:500, NBP221578, Novus, USA), followed by horseradish peroxidaseconjugated goat anti-rabbit antibody (Jackson Immunoresearch Laboratories, USA). Protein signals were visualized using enhanced chemiluminescence assay kit (Thermo Scientific, USA). Bands intensities were quantified using Image J, a java-based image processing program.

\section{Statistics Analysis}

Statistical analyses was conducted using SPSS v. 22 for Windows (IBM, NY, USA). One or 2-way ANOVA followed by post hoc test was applied on normally distributed data set. For data which were not normally distributed, Wilcoxon signed-rank and KruskalWallis tests were used. Pearson and Spearman correlations were used for parametric and non-parametric variables. All numerical data are expressed as mean \pm SEM, unless otherwise indicated. $p$-Values of $<0.05$ were considered significant.

\section{RESULTS}

\section{Temporal and Spatial Profile of Thrombin Activity in Whole Slices following pMCAo}

The pMCAo procedure in 11 mice resulted in consistent but moderate neurological deficits in all groups with a trend for more severe outcome in the $24 \mathrm{~h}$ group (median $=0,2,2,3$ for control mice and mice that underwent 2,5 , and $24 \mathrm{~h}$ pMCAo respectively; $p=0.001$, by Kruskal-Wallis test). In order to determine the temporal profile of thrombin activity in mouse brain following a focal ischemic event, we measured brain thrombin activity levels 2, 5, and $24 \mathrm{~h}$ following pMCAo (Table S1 in Supplementary Material).

Figure 1 presents the mean thrombin activity levels in the ischemic and contralateral hemispheres at various time intervals after pMCAo. A repeated measures model was used to assess the association between slice number, occlusion time (control, 2,5 , and $24 \mathrm{~h}$ ), the interaction between the two, and thrombin activity. In the anterior and middle parts of the ischemic hemisphere (slices 3-8 right), a significant effect was found for slice number $[F(1.150,4.598)=76.584, p=0.004]$ and occlusion time $[F(1.726,6.904)=13.826, p=0.0004]$ with a significant interaction between the two $[F(2.090,8.360)=7.271, p=0.014]$. In a post hoc analysis, the source of the difference between slice numbers was from the comparison of slices $3,4,5,7$, and 8 to slice 6 $(p=0.002, p=0.016, p=0.067, p=0.009, p=0.015)$. In addition, the source of the difference between occlusion times was from the comparison of control with the stroke groups $(p=0.047$; $p=0.041 ; p=0.001$ for 2,5 and $24 \mathrm{~h}$, respectively). These findings reflect a substantial increase in thrombin activity at $24 \mathrm{~h}$ 


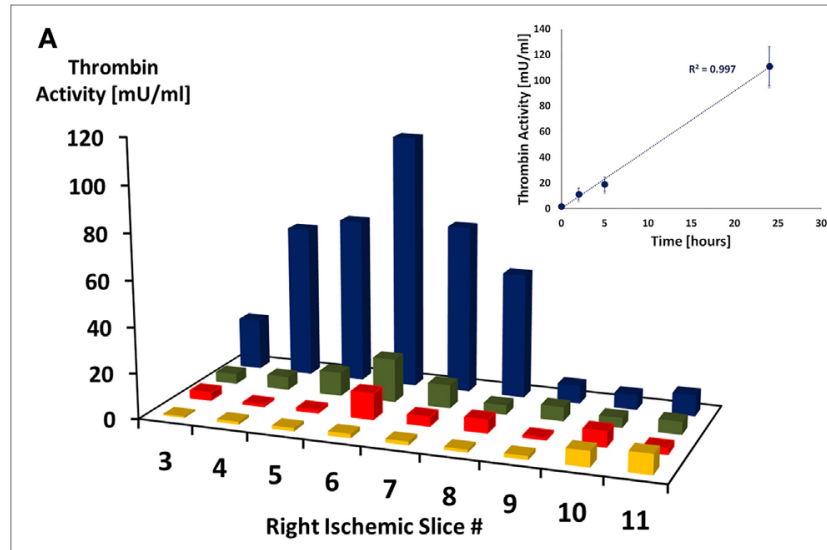

B

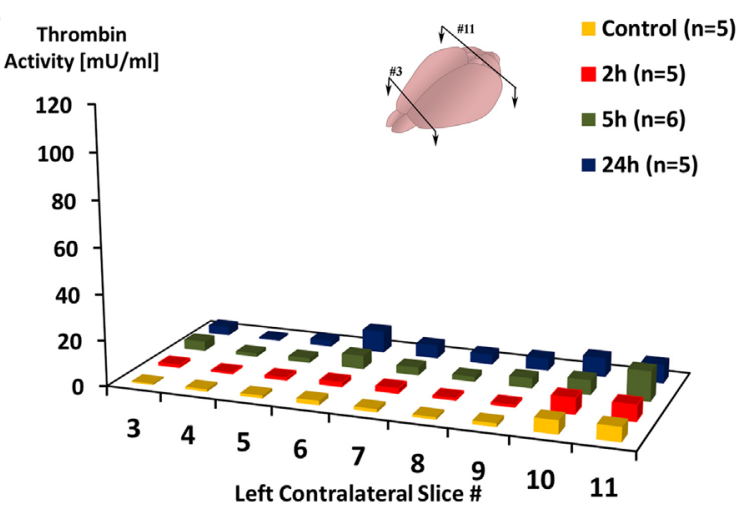

FIGURE 1 | Thrombin activity in the ischemic hemisphere following permanent middle cerebral artery occlusion. Mean thrombin activity levels measured in brain slices taken from the right ischemic (A) and left contralateral (B) hemispheres, at the indicated time intervals after right permanent MCAo. Slices were numbered from anterior (\#3) to posterior (\#11), slices' thickness $=1 \mathrm{~mm}$. Inset is a plot of the mean thrombin activity levels that were measured in slice \#6 as function of occlusion time. $n$ represent number of mice that were used in each group.

compared to $5 \mathrm{~h}$ post ischemia $(p=0.001)$, as clearly evident from Figure 1A. Strikingly, a linear correlation was found between thrombin activity levels that were measured in the ischemic core (slice \#6) to occlusion time ( $r=0.998, p=0.001$ by Pearson correlation; inset in Figure 1A). In contrast to the ischemic hemisphere, in the anterior and middle parts of the contralateral hemisphere (slices 3-8 left), although there was a moderate effect for slice number $[F(1.888,7.552)=8.241, p=0.013]$, no significant effect was found for occlusion time $(p=0.358)$ nor for the interaction between the two ( $p=0.433$, Figure 1B). At all time points, thrombin activity levels in the ischemic hemisphere were higher compared to the contralateral hemisphere $\{2 \mathrm{~h}$ $[F(1.000,4.000)=21.908, p=0.009] ; 5 \mathrm{~h}[F(1.000,5.000)=4.934$, $p=0.077] ; 24 \mathrm{~h}[F(1.000,4.000)=42.096, p=0.003]\}$. In the control group, no significant differences was found between the two hemispheres $(p=0.57)$. In the posterior part of the ischemic hemisphere (slices 9-11), thrombin activity was not significantly changed. No significant effect was found for either for slice number $(p=0.821)$ or for occlusion time $(p=0.278)$ in these posterior slices.

\section{Spatial Distribution of Thrombin Activity in Brain Slice}

Since thrombin levels were maximally elevated $24 \mathrm{~h}$ following stroke induction, we further evaluated the spatial distribution of this activity by obtaining punch samples from mice $(n=4)$ which underwent pMCAo at this time point. The localization of the standard punch samples was from the core area of the ischemic damage in the basal ganglia and in the cortical area corresponding to the penumbra (40-42). These areas (C,D, respectively) and corresponding areas in the contralateral hemisphere $(A, B)$ are marked out in a representative slice stained for ischemic damage by TTC as presented in Figure 2A. The highest thrombin activity levels were measured in the basal ganglia of the ischemic hemisphere, and they were higher compared to those measured in the ischemic cortex $(271 \pm 117$ vs. $122 \pm 27 \mathrm{mU} / \mathrm{ml} ; n=4 ; p=0.072$, by Wilcoxon signed-rank test; Figure 2B). As expected, these thrombin activity values that were measured in both the cortex and the basal ganglia of the ischemic hemisphere were higher compared to the corresponding areas in the contralateral slices $(122 \pm 27,271 \pm 117$, vs. $3 \pm 3,10 \pm 6 \mathrm{mU} / \mathrm{ml} ; n=4 ; p=0.039$ and 0.039 , respectively, by Wilcoxon signed-rank test; Figure 2B).

The distribution of thrombin activity in the core vs. surrounding areas was further evaluated using a fluorescence histochemical method. Figures 2C-E are representative slides obtained from one animal out of five examined and presents the topographic distribution of thrombin activity $24 \mathrm{~h}$ following pMCAo. High density of small, discrete, yellow-orange fluorescent needle shaped crystals that mark the locations of thrombin activity (Figure 2F) were observed in the right ischemic hemisphere in the cortical areas and in the basal ganglia compared to lower thrombin activity contralaterally (Figure 2C). The histochemical localization of thrombin activity corresponds to the infarct areas per TTC staining and to areas with high thrombin activity levels as determined using the thrombin activity assay and tissue punches technique (Figures 2A,B). Interestingly, higher thrombin activity levels were observed in the deep layers of the ischemic parietal cortex compared to lower thrombin activity in its upper layers (Figures 2C-E). No staining was seen in the corpus callosum of the ischemic hemisphere (Figures 2C-E) and in the sections that were incubated with the thrombin inhibitor NAPAP (Figure 2G).

\section{PAR1 and EPCR Levels following pMCAo}

Figure 3 presents levels of PAR1 and EPCR in the ischemic hemispheres relative to the contralateral hemispheres measured by western blot technique at 2,5 , and $24 \mathrm{~h}$ following pMCAo. In parallel to the increase in thrombin activity in the ischemic hemisphere, PAR1 levels decreased compared to the contralateral area upon MCAo. Following $5 \mathrm{~h}$ of pMCAo, the ratio of PAR1 levels in the ischemic core to contralateral areas was lower compared to control mice (Figure 3A, $p=0.055$, by Kruskal-Wallis test). This decrease was further augmented following $24 \mathrm{~h}$ of pMCAo $(p=0.004$; Figure 3A). There was a significant inverse correlation between thrombin activity and PAR1 level as can be seen in Figure $4(r=-0.491, p=0.037)$. Similarly, the ratio of EPCR 

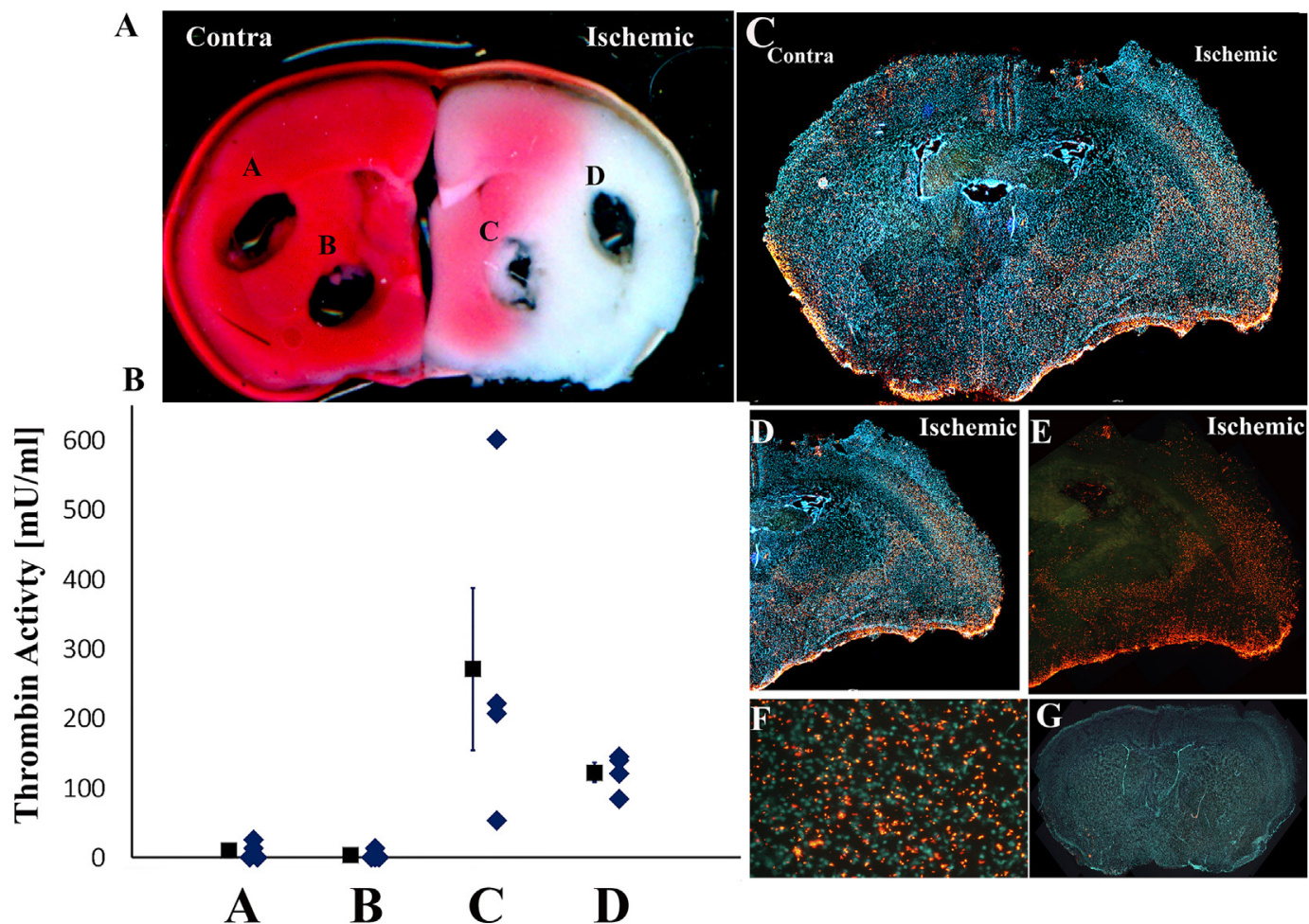

FIGURE 2 | Spatial distribution of thrombin activity in brain slice. (A) The locations of the 1.5-mm diameter tissue punches sampled from fresh slices (slice location $1 \mathrm{~mm}$ posterior to the bregma; slice \#6 in Figure 1). Typical triphenyltetrazolium chloride staining of the relevant slice used in these analyses is presented (representative of four slices developed by this method). Infarct regions are colored by white and intact brain regions by red. (B) Mean thrombin activity levels (milliunits per milliliter of tissue \pm SEM) and its data distribution that were measured at the cortex and basal ganglia in the ischemic and contralateral hemispheres following permanent middle cerebral artery occlusion (pMCAo). (C-E) Fluorescence photomicrograph of coronal sections of mice brains $24 \mathrm{~h}$ following pMCAo that were incubated with Boc-Asp(OBzl)-Pro-Arg-4MBNA. A mosaic was formed by merging 20 pictures, each picture magnification 40x (C). The small, discrete, yellow-orange fluorescent, needle shaped crystals represent the locations of thrombin activity. Cell nuclei were stained by hoechst and appear as blue spots (C,D). (F) Higher-power photomicrograph of the typical appearance and distribution of the thrombin activity reaction product in the cortical, basal ganglia areas in the ischemic hemispheres (magnification 200x). (G) Absence of thrombin activity reaction product in tissue incubated in histochemical staining solution containing the specific thrombin inhibitor NAPAP. A mosaic was formed by merging 20 pictures, each picture magnification 40x.
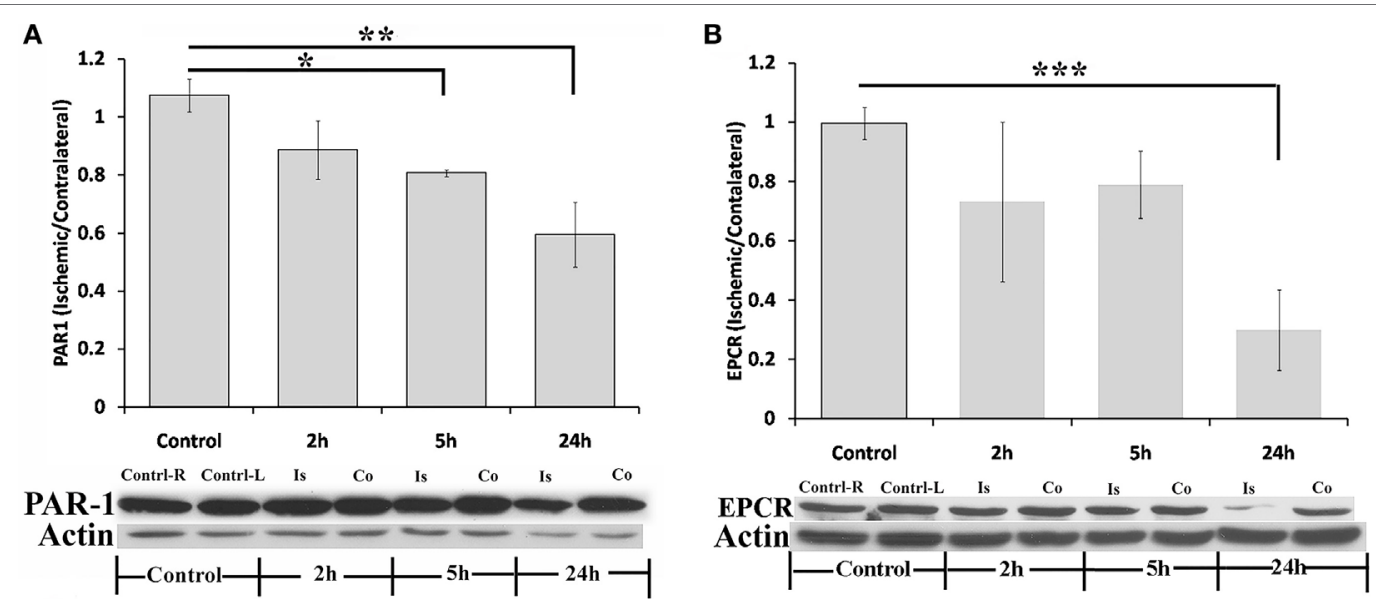

FIGURE $3 \mid$ Protease-activated receptor 1 (PAR1) and endothelial cell protein C receptor (EPCR) levels decrease following permanent middle cerebral artery occlusion (pMCAo). Ratio of PAR1 (A) and EPCR (B) levels in the ischemic core vs. their levels in corresponding areas in the contralateral hemisphere, as measured at various times intervals after right pMCAo $\left({ }^{*} p=0.055,{ }^{\star \star} p=0.004\right.$, by Kruskal-Wallis test, $n=5 ;{ }^{{ }^{* \star}} p=0.047$, by one-way ANOVA following post hoc Tukey's test, $n=4$;). Is = Ischemic; Co = contralateral. 


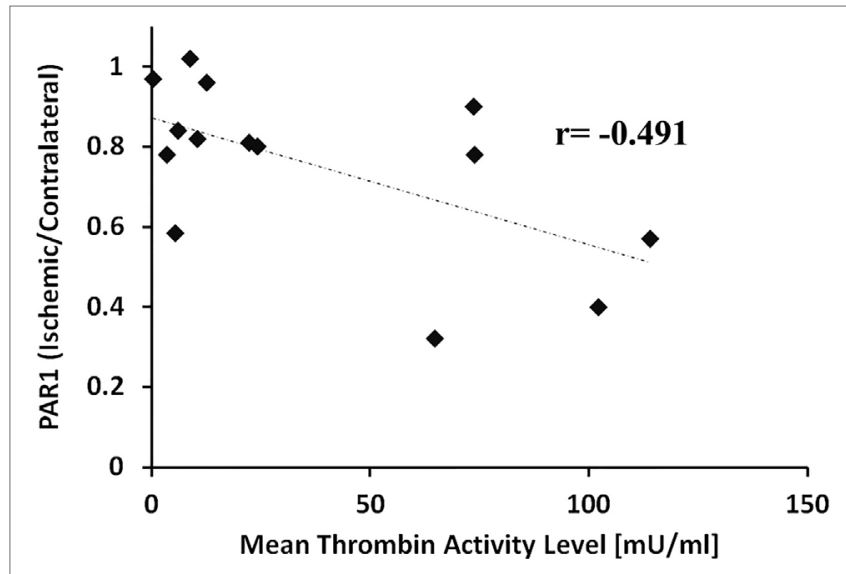

FIGURE 4 | Negative correlation between thrombin activity and protease-activated receptor 1 (PAR1) levels. Ratio of PAR1 levels in the ischemic core vs. the corresponding areas in the contralateral hemisphere as a function of the mean thrombin activity measured in the ischemic core (slices \# 5, 6, 7) 2, 5, and $24 \mathrm{~h}$, following permanent middle cerebral artery occlusion. The correlation was significant by Spearman's test $(r=-0.491$, $p=0.037)$.

levels in the ischemic core to the contralateral areas decreased with time after pMCAo, and this was lower compared to control mice $24 \mathrm{~h}$ following pMCAo ( $p=0.047$ by one-way ANOVA following post hoc Tukey's test; Figure 3B).

\section{DISCUSSION}

In the present study, we found that thrombin activity in the ischemic core started to rise $2 \mathrm{~h}$ after MCAo, and activity levels continued to rise and to expand into additional areas that surround the ischemic core. Twenty-four hours after MCAo, levels of thrombin activity rose dramatically in the anterior and middle regions of the ischemic hemisphere reaching peak levels in the ischemic core. Strikingly, thrombin activity levels in the ischemic core rose linearly over time. In contrast, thrombin activity in slices that were taken from areas that are supplied by the posterior circulation (\#9-11), and in the contralateral hemisphere remained as expected unchanged. Levels of thrombin activity that were measured using discrete tissue samples taken from the ischemic core reached values that are more than two times higher compared to those obtained when sampling the entire hemisphere. It is likely that the levels found when sampling the entire hemisphere are an underestimation and represent an average between higher levels in the infarct core and lower levels in perinfarct areas. The propagation of the thrombin activity across slices suggests a time-dependent expansion of the core of thrombin activity.

Previously, we reported that thrombin concentrations above $60 \mathrm{mU} / \mathrm{ml}$ are able to alter synaptic responses by increasing paired pulse facilitation as well as network excitability leading to synaptic dysfunction (39). In this study, we found a linear increase of thrombin activity and that $24 \mathrm{~h}$ following occlusion, thrombin activity in the ischemic hemisphere is considerably above the deleterious threshold. In contrast, thrombin activity levels that were measured in the ischemic core $5 \mathrm{~h}$ following MCAo were below the deleterious limit of $60 \mathrm{mU} / \mathrm{ml}$. Interestingly, this time frame fits the therapeutic window for effective reperfusion therapy (43).

In the current study, thrombin activity levels that were measured in the ischemic core $24 \mathrm{~h}$ following pMCAo were higher compared to those measured in a previous study of ours (12). The reason for these differences is probably due to the different types of filament that were used to occlude the MCA in each study (commercial silicone-coated filament 4-mm length compared to heat-blunted 6-0 suture coated with poly-L-lysine). Indeed, Guan et al. showed in a MCAo model performed in rats, positive correlation between suture coating length and infarct volume (44). Likewise, our group has found a positive correlation between infarcts volume to thrombin activity levels $(12,39)$. Not surprisingly, thrombin activity levels that were measured in the ischemic core $24 \mathrm{~h}$ following $90 \mathrm{~min}$ tMCAo (39) were lower compared to those measured following pMCAo. It is expected that in the transient model, most of the blood vessels will undergo reperfusion in such a situation and that there will be less thrombin activity left over from the initial tissue damage as well as less propagation of thrombin activity due to tissue ischemia and continued occlusion of blood vessels. In contrast to our previous study (39), in this study, we present mapping of brain thrombin activity levels following pMCAo, at several time points from the ischemic event and not just $24 \mathrm{~h}$ later.

The specific time points that we used in this study represent critical stages in the progression of stroke: $2 \mathrm{~h}$-acute stage, immediately after the onset; $5 \mathrm{~h}$-the end of the therapeutic window; $24 \mathrm{~h}$-peak point of thrombin activity following global ischemia (45) and optimal time point for TTC staining. The temporal profile of thrombin activity that is presented in this study is comparable to that found following global cerebral ischemia that was performed using bilateral common carotid artery occlusion (BCCAO) (45). Following BCCAO, brain thrombin activity slightly increased at $4 \mathrm{~h}$, peaked at $24 \mathrm{~h}$, and declined at $72 \mathrm{~h}$. The rapid increase of thrombin activity in the first hours is compatible with the findings that thrombin activity positively correlates to infarct volume $(12,39)$ and to the very early appearance of ischemic lesion that was observed following pMCAo (2).

The highest thrombin activity levels measured biochemically in tissue punches were found in infarct areas based on TTC staining. This finding is in agreement with our previous finding of a positive correlation between thrombin activity level and infarct size $(12,39)$. Furthermore, we have found that the distribution of thrombin activity in the tested slices was similar in both the punch method and the new higher resolution histochemical method. In both methods, high thrombin activity levels were observed in the right ischemic hemisphere in the cortical areas and in the basal ganglia compared to negligible thrombin activity levels contralaterally. Interestingly, non-homogeneous distribution of thrombin activity was observed in the cortical layers of the ischemic hemisphere with higher activity in the deep layers. Furthermore, no staining of thrombin activity was seen in the corpus callosum of the ischemic hemisphere. Further experiments are needed in order to better clarify these spatial patterns. 
In parallel with the linear increase of thrombin activity, PAR1 levels in the ischemic core decreased as stroke progressed. These results are in agreement with the study of Chen et al. that found decrease in PAR1 levels in rat brains in the first hours after tMCAo (13). In contrast, PAR1 levels were raised in rat hippocampal slice cultures that were subject to experimental ischemia [oxygen-glucose deprivation (OGD)] (46) and in mice brains undergoing global cerebral ischemia (45). A possible explanation for this apparent conflict is that in the MCAo model, high level of thrombin activity that originates in the blood stream (13), enters into the brain tissue through vascular disruption, and activates PAR1 on cell membranes. Consequently, PAR1 rapidly internalize and degrade. The negative correlation observed between thrombin activity in the ischemic core to the ratio of PAR1 levels in the ischemic core vs. the contralateral hemisphere emphasizes the link between PAR1 reduction and elevated thrombin activity. In contrast, in brain slices that are subjected to ischemic conditions such as OGD, no circulating coagulation factors are involved and the time points examined were somewhat shorter. Thrombin that is generated in the brain cells rises $(14,15)$ and as a result PAR1, its own receptor, may first increase as a complementary step, but these receptors are then cleaved and internalized leading to reduced levels. It is hypothesized that inhibiting the levels of thrombin during acute stroke will normalize PAR1 levels at the 2-24 hour time frame.

Several studies have demonstrated the neuroprotective effects of deletion, inhibition or silencing PAR1 in brain ischemia $(13,20$, 47-49). In a previous study we have shown that pharmacological inhibition of either thrombin or PAR1, restores physiological synaptic plasticity that was blocked in OGD neuronal networks. Moreover, we have found that hippocampal slices prepared from PAR1-KO mice that were exposed to OGD have normal synaptic transmission (15). In the current study we found that PAR1 levels in the ischemic core start to decrease $5 \mathrm{~h}$ following MCAo, as a results it is likely that treatment using PAR1 inhibitors might be effective only if administered in the first hours from the ischemic event.

Endothelial cell protein $\mathrm{C}$ receptor decreased in the ischemic core only at a late phase, $24 \mathrm{~h}$ following pMCAo. Proteinase 3 (PR3) a neutrophil granule proteinase is elevated in the brain following stroke and is involved in the inflammatory process (50). Villegas-Mendez et al found high affinity interaction between the neutrophil protease PR3, and the EPCR, which results in the proteolytic degradation of the receptor (51). Degradation of EPCR with consequent loss of aPC generation is likely to contribute to the already known proinflammatory roles of PR3 (51).

The novelty of the present study is to show for the first time quantitative characterization of the temporal and spatial profiles of brain thrombin activity during the course of acute ischemic stroke. The results reveals continuous increase and spatial expansion of thrombin activity in mice brains following MCAo even in areas outside the ischemic core. Furthermore, changes in PAR1 and EPCR levels during stroke progress are presented. The potential translation of these results into the clinic is by consideration of pharmacologically inhibiting thrombin activity in the brain early after an acute ischemic event as an optional complementary therapy for acute stroke in order to prevent secondary thrombin related brain damage. However, due to the continuous increase of thrombin activity, the therapeutic dose of the thrombin inhibitor should be time dependent. In contrast to thrombin activity, the decrease of PAR1 during stroke progress suggests that PAR1 inhibition might be not affective if it will be given in time delay from the ischemic event.

The main limitation of this study is that the experiments were performed only on healthy young male mice. In order to translate the results of this study to clinical implementation, future studies should include old as well as female mice. In addition, in future experiments, it will be interesting to study the role of thrombin activity in the transient occlusion model that simulates reperfusion of the artery.

In summary, findings of this study underscore the temporal and spatial profile of brain thrombin activity during the course of acute ischemic stroke. The dramatic increase over time in brain thrombin activity is in agreement with the therapeutic window for reperfusion therapy and the known progression of brain damage and may constitute a potential complimentary target for acute ischemic stroke therapy.

\section{ETHICS STATEMENT}

This study was carried out in accordance with the recommendations of the Institutional Animal Care and Use Committee of The Chaim Sheba Medical Center (Tel HaShomer, Israel), which adheres to the Israeli law on the use of laboratory animals and NIH rules. The protocol was approved by the Institutional Animal Care and Use Committee of Tel HaShomer.

\section{AUTHOR CONTRIBUTIONS}

DB designed the study, performed the experiments (thrombin activity, stroke model), analyzed data and wrote the paper. OG and EF helped with the western blot experiments and data analysis. ES and VG helped with the prepared sections for staining. JC and DT supervised the project and revised the manuscript critically for important intellectual content. All authors gave their approval to the manuscript.

\section{ACKNOWLEDGMENTS}

This work was performed in partial fulfillment of the requirements for a Ph.D. degree by Doron Bushi at the Sackler Faculty of Medicine, Tel Aviv University, Israel.

\section{FUNDING}

The research was supported by institutional funding.

\section{SUPPLEMENTARY MATERIAL}

The Supplementary Material for this article can be found online at http://journal.frontiersin.org/article/10.3389/fneur.2017.00138/ full\#supplementary-material. 


\section{REFERENCES}

1. Saver JL. Time is brain - quantified. Stroke (2006) 37(1):263-6. doi:10.1161/ 01.STR.0000196957.55928.ab

2. Garcia JH, Yoshida Y, Chen H, Li Y, Zhang ZG, Lian J, et al. Progression from ischemic injury to infarct following middle cerebral artery occlusion in the rat. Am J Pathol (1993) 142(2):623-35.

3. Verheugt FW, Granger CB. Oral anticoagulants for stroke prevention in atrial fibrillation: current status, special situations, and unmet needs. Lancet (2015) 386:303-10. doi:10.1016/S0140-6736(15)60245-8

4. Maggio N, Blatt I, Vlachos A, Tanne D, Chapman J, Segal M. Treating seizures and epilepsy with anticoagulants? Front Cell Neurosci (2013) 7:19. doi:10.3389/ fncel.2013.00019

5. Bar-Shavit R, Hruska KA, Kahn AJ, Wilner GD. Hormone-like activity of human thrombin. Ann N Y Acad Sci (1986) 485:335-48. doi:10.1111/j. 1749-6632.1986.tb34595.x

6. Coughlin SR. Thrombin signalling and protease-activated receptors. Nature (2000) 407(6801):258-64. doi:10.1038/35025229

7. Xi G, Reiser G, Keep RF. The role of thrombin and thrombin receptors in ischemic, hemorrhagic and traumatic brain injury: deleterious or protective? J Neurochem (2003) 84(1):3-9. doi:10.1046/j.1471-4159.2003.01268.x

8. Noorbakhsh F, Vergnolle N, Hollenberg MD, Power C. Proteinase-activated receptors in the nervous system. Nat Rev Neurosci (2003) 4(12):981-90. doi:10.1038/nrn1255

9. Xue M, Del Bigio MR. Acute tissue damage after injections of thrombin and plasmin into rat striatum. Stroke (2001) 32(9):2164-9. doi:10.1161/hs0901. 095408

10. Kameda K, Kikkawa Y, Hirano M, Matsuo S, Sasaki T, Hirano K. Combined argatroban and anti-oxidative agents prevents increased vascular contractility to thrombin and other ligands after subarachnoid haemorrhage. Br J Pharmacol (2012) 165(1):106-19. doi:10.1111/j.1476-5381.2011.01485.x

11. Chen B, Cheng Q, Yang K, Lyden PD. Thrombin mediates severe neurovascular injury during ischemia. Stroke (2010) 41(10):2348-52. doi:10.1161/ STROKEAHA.110.584920

12. Bushi D, Chapman J, Katzav A, Shavit-Stein E, Molshatzki N, Maggio N, et al. Quantitative detection of thrombin activity in an ischemic stroke model. J Mol Neurosci (2013) 51(3):844-50. doi:10.1007/s12031-013-0072-y

13. Chen B, Friedman B, Whitney MA, Winkle JA, Lei IF, Olson ES, et al. Thrombin activity associated with neuronal damage during acute focal ischemia. J Neurosci (2012) 32(22):7622-31. doi:10.1523/JNEUROSCI.0369-12.2012

14. Thevenet J, Angelillo-Scherrer A, Price M, Hirt L. Coagulation factor Xa activates thrombin in ischemic neural tissue. J Neurochem (2009) 111(3): 828-36. doi:10.1111/j.1471-4159.2009.06369.x

15. Stein ES, Itsekson-Hayosh Z, Aronovich A, Reisner Y, Bushi D, Pick CG, et al. Thrombin induces ischemic LTP (iLTP): implications for synaptic plasticity in the acute phase of ischemic stroke. Sci Rep (2015) 5:7912. doi:10.1038/ srep07912

16. Becker D, Ikenberg B, Schiener S, Maggio N, Vlachos A. NMDA-receptor inhibition restores protease-activated receptor 1 (PAR1) mediated alterations in homeostatic synaptic plasticity of denervated mouse dentate granule cells. Neuropharmacology (2014) 86:212-8. doi:10.1016/j.neuropharm.2014.07.013

17. Maggio N, Cavaliere C, Papa M, Blatt I, Chapman J, Segal M. Thrombin regulation of synaptic transmission: implications for seizure onset. Neurobiol Dis (2013) 50:171-8. doi:10.1016/j.nbd.2012.10.017

18. Maggio N, Itsekson Z, Dominissini D, Blatt I, Amariglio N, Rechavi G, et al. Thrombin regulation of synaptic plasticity: implications for physiology and pathology. Exp Neurol (2013) 247:595-604. doi:10.1016/j.expneurol. 2013.02.011

19. Maggio N, Shavit E, Chapman J, Segal M. Thrombin induces long-term potentiation of reactivity to afferent stimulation and facilitates epileptic seizures in rat hippocampal slices: toward understanding the functional consequences of cerebrovascular insults. J Neurosci (2008) 28(3):732-6. doi:10.1523/ JNEUROSCI.3665-07.2008

20. Hamill CE, Mannaioni G, Lyuboslavsky P, Sastre AA, Traynelis SF. Proteaseactivated receptor 1-dependent neuronal damage involves NMDA receptor function. Exp Neurol (2009) 217(1):136-46. doi:10.1016/j.expneurol.2009.01.023

21. Suo Z, Citron BA, Festoff BW. Thrombin: a potential proinflammatory mediator in neurotrauma and neurodegenerative disorders. Curr Drug Targets Inflamm Allergy (2004) 3(1):105-14. doi:10.2174/1568010043483953
22. Junge CE, Sugawara T, Mannaioni G, Alagarsamy S, Conn PJ, Brat DJ, et al. The contribution of protease-activated receptor 1 to neuronal damage caused by transient focal cerebral ischemia. Proc Natl Acad Sci U S A (2003) 100(22):13019-24. doi:10.1073/pnas.2235594100

23. Lyden P, Pereira B, Chen B, Zhao L, Lamb J, Lei IF, et al. Direct thrombin inhibitor argatroban reduces stroke damage in 2 different models. Stroke (2014) 45(3):896-9. doi:10.1161/STROKEAHA.113.004488

24. Morris DC, Zhang L, Zhang ZG, Lu M, Berens KL, Brown PM, et al. Extension of the therapeutic window for recombinant tissue plasminogen activator with argatroban in a rat model of embolic stroke. Stroke (2001) 32(11):2635-40. doi:10.1161/hs1101.097390

25. Kate M, Gioia L, Buck B, Sivakumar L, Jeerakathil T, Shuaib A, et al. Dabigatran therapy in acute ischemic stroke patients without atrial fibrillation. Stroke (2015) 46(9):2685-7. doi:10.1161/STROKEAHA.115.010383

26. Tomita H, Hagii J, Metoki N, Saito S, Shiroto H, Hitomi H, et al. Severity and functional outcome of patients with cardioembolic stroke occurring during non-vitamin $\mathrm{K}$ antagonist oral anticoagulant treatment. J Stroke Cerebrovasc Dis (2015) 24(6):1430-7. doi:10.1016/j.jstrokecerebrovasdis.2015.03.004

27. Yang-Hyun L, Tae-Won K, Hyun-Ji C, Jeong-Wook P, Kwang-Soo L. Therapeutic effect and safety of argatroban in cerebral territory infarction. J Neurocrit Care (2016) 9:132-8. doi:10.18700/jnc.160067

28. Morrow DA, Alberts MJ, Mohr JP, Ameriso SF, Bonaca MP, Goto S, et al. Efficacy and safety of vorapaxar in patients with prior ischemic stroke. Stroke (2013) 44(3):691-8. doi:10.1161/STROKEAHA.111.000433

29. Van de Wouwer M, Collen D, Conway EM. Thrombomodulin-protein C-EPCR system: integrated to regulate coagulation and inflammation. Arterioscler Thromb Vasc Biol (2004) 24(8):1374-83. doi:10.1161/01.ATV.0000134298. 25489.92

30. Cheng T, Liu D, Griffin JH, Fernandez JA, Castellino F, Rosen ED, et al. Activated protein C blocks p53-mediated apoptosis in ischemic human brain endothelium and is neuroprotective. Nat Med (2003) 9(3):338-42. doi:10.1038/ nm826

31. Domotor E, Benzakour O, Griffin JH, Yule D, Fukudome K, Zlokovic BV. Activated protein $\mathrm{C}$ alters cytosolic calcium flux in human brain endothelium via binding to endothelial protein $\mathrm{C}$ receptor and activation of protease activated receptor-1. Blood (2003) 101(12):4797-801. doi:10.1182/ blood-2002-12-3680

32. Mosnier LO, Zlokovic BV, Griffin JH. Cytoprotective-selective activated protein C therapy for ischaemic stroke. Thromb Haemost (2014) 112(5):883-92. doi:10.1160/TH14-05-0448

33. Riewald M, Petrovan RJ, Donner A, Mueller BM, Ruf W. Activation of endothelial cell protease activated receptor 1 by the protein C pathway. Science (2002) 296(5574):1880-2. doi:10.1126/science.1071699

34. Strande JL, Hsu A, Su J, Fu X, Gross GJ, Baker JE. SCH 79797, a selective PAR1 antagonist, limits myocardial ischemia/reperfusion injury in rat hearts. Basic Res Cardiol (2007) 102(4):350-8. doi:10.1007/s00395-007-0653-4

35. Hamill CE, Caudle WM, Richardson JR, Yuan H, Pennell KD, Greene JG, et al. Exacerbation of dopaminergic terminal damage in a mouse model of Parkinson's disease by the G-protein-coupled receptor protease-activated receptor 1. Mol Pharmacol (2007) 72(3):653-64. doi:10.1124/mol.107.038158

36. Isaev D, Lushnikova I, Lunko O, Zapukhliak O, Maximyuk O, Romanov A, et al. Contribution of protease-activated receptor 1 in status epilepticusinduced epileptogenesis. Neurobiol Dis (2015) 78:68-76. doi:10.1016/j. nbd.2015.03.026

37. Longa EZ, Weinstein PR, Carlson S, Cummins R. Reversible middle cerebral artery occlusion without craniectomy in rats. Stroke (1989) 20(1):84-91. doi:10.1161/01.STR.20.1.84

38. Bushi D, Gera O, Kostenich G, Shavit-Stein E, Weiss R, Chapman J, et al. A novel histochemical method for the visualization of thrombin activity in the nervous system. Neuroscience (2016) 320:93-104. doi:10.1016/j. neuroscience.2016.01.065

39. Bushi D, Ben Shimon M, Shavit Stein E, Chapman J, Maggio N, Tanne D. Increased thrombin activity following reperfusion after ischemic stroke alters synaptic transmission in the hippocampus. J Neurochem (2015) 135(6): 1140-8. doi:10.1111/jnc. 13372

40. Hossmann KA. Cerebral ischemia: models, methods and outcomes. Neuropharmacology (2008) 55(3):257-70. doi:10.1016/j.neuropharm.2007.12.004

41. Olah L, Wecker S, Hoehn M. Relation of apparent diffusion coefficient changes and metabolic disturbances after 1 hour of focal cerebral ischemia 
and at different reperfusion phases in rats. J Cereb Blood Flow Metab (2001) 21(4):430-9. doi:10.1097/00004647-200104000-00012

42. Hossmann KA. The two pathophysiologies of focal brain ischemia: implications for translational stroke research. J Cereb Blood Flow Metab (2012) 32(7):1310-6. doi:10.1038/jcbfm.2011.186

43. Goyal M, Menon BK, van Zwam WH, Dippel DW, Mitchell PJ, Demchuk AM, et al. Endovascular thrombectomy after large-vessel ischaemic stroke: a meta-analysis of individual patient data from five randomised trials. Lancet (2016) 387(10029):1723-31. doi:10.1016/S0140-6736(16)00163-X

44. Guan Y, Wang Y, Yuan F, Lu H, Ren Y, Xiao T, et al. Effect of suture properties on stability of middle cerebral artery occlusion evaluated by synchrotron radiation angiography. Stroke (2012) 43(3):888-91. doi:10.1161/STROKEAHA. 111.636456

45. Wang J, Jin H, Hua Y, Keep RF, Xi G. Role of protease-activated receptor-1 in brain injury after experimental global cerebral ischemia. Stroke (2012) 43(9):2476-82. doi:10.1161/STROKEAHA.112.661819

46. Striggow F, Riek-Burchardt M, Kiesel A, Schmidt W, Henrich-Noack P, Breder J, et al. Four different types of protease-activated receptors are widely expressed in the brain and are up-regulated in hippocampus by severe ischemia. Eur J Neurosci (2001) 14(4):595-608. doi:10.1046/j.0953-816x.2001.01676.x

47. Junge CE, Lee CJ, Hubbard KB, Zhang Z, Olson JJ, Hepler JR, et al. Proteaseactivated receptor-1 in human brain: localization and functional expression in astrocytes. Exp Neurol (2004) 188(1):94-103. doi:10.1016/j.expneurol. 2004.02.018

48. Rajput PS, Lyden PD, Chen B, Lamb JA, Pereira B, Lamb A, et al. Protease activated receptor- 1 mediates cytotoxicity during ischemia using in vivo and in vitro models. Neuroscience (2014) 281:229-40. doi:10.1016/j.neuroscience. 2014.09.038

49. Zhang J, Wang Y, Zhu P, Wang X, Lv M, Feng H. siRNA-mediated silence of protease-activated receptor-1 minimizes ischemic injury of cerebral cortex through HSP70 and MAP2. J Neurol Sci (2012) 320(1-2):6-11. doi:10.1016/j. jns.2012.05.040

50. Jickling GC, Liu D, Ander BP, Stamova B, Zhan X, Sharp FR. Targeting neutrophils in ischemic stroke: translational insights from experimental studies. J Cereb Blood Flow Metab (2015) 35(6):888-901. doi:10.1038/jcbfm. 2015.45

51. Villegas-Mendez A, Montes R, Ambrose LR, Warrens AN, Laffan M, Lane DA. Proteolysis of the endothelial cell protein $\mathrm{C}$ receptor by neutrophil proteinase 3. J Thromb Haemost (2007) 5(5):980-8. doi:10.1111/j. 1538-7836.2007.02480.x

Conflict of Interest Statement: The authors declare that the research was conducted in the absence of any commercial or financial relationships that could be construed as a potential conflict of interest.

Copyright (c) 2017 Bushi, Stein, Golderman, Feingold, Gera, Chapman and Tanne. This is an open-access article distributed under the terms of the Creative Commons Attribution License (CC BY). The use, distribution or reproduction in other forums is permitted, provided the original author(s) or licensor are credited and that the original publication in this journal is cited, in accordance with accepted academic practice. No use, distribution or reproduction is permitted which does not comply with these terms. 